\title{
El lugar de la cultura en la era del capitalismo cognitivo. Notas para una discusión sobre ciudadanía digital*
}

\author{
The location of culture in cognitive capitalism \\ era. Notes for a discussion on digital citizenship
}

\section{RESUMEN:}

La cultura tiene un rol fundamental como motor de cambio social y en los procesos de desarrollo. Ahora bien, en el capitalismo cognitivo, la mercantilización de lo inmaterial restringe la diversidad y el pluralismo, homogeneiza imaginarios y anula tradiciones populares bajo la presión de un mono-discurso hegemónico y totalizador. Los países del sur, las expresiones de las minorías o las prácticas mediáticas alternativas, por ejemplo, quedan silenciadas o desplazadas a un segundo plano, impidiendo el desarrollo de los pobres, los desplazados o los diferentes. No obstante, las Nuevas Tecnologías de la Información y la Comunicación (NTIC's) ofrecen un nuevo escenario donde la ciudadanía puede incrementar la participación y el empoderamiento local, claves para la salud de la democracia. $Y$ es que el capital informacional que Internet aporta en lo que a participación y cooperación ciudadana se refiere es esencial para el desarrollo y el cambio social. Ahora bien, estos procesos de apropiación de las NTIC's, por parte de la ciudadanía, están mediados por una serie de ejes de tensión o dialécticas. Para trascender estas dificultades que el entorno digital plantea sobre la democracia es necesario buscar modelos teórico-metodológicos de la mediación compleja que abran espacios públicos y permitan el empoderamiento local de la ciudadanía. En este sentido, se proponen una serie de líneas de actuación para elaborar políticas de comunicación y cultura que contribuyan al desarrollo de una ciudadanía digital activa y participativa.

\section{PALABRAS CLAVE:}

Cultura; Participación; Ciudadanía digital; Políticas de comunicación; NTIC's; Desarrollo; Cambio social.

\footnotetext{
* El presente texto forma parte del Proyecto de I+D «Ciberactivismo, ciudadanía digital y nuevos movimientos urbanos" (CiberMov), coordinado por el Grupo Interdisciplinario de Estudios en Comunicación, Política y Cambio Social (COMPOLÍTICAS) de la Universidad de Sevilla y financiado por el Programa Estatal de Fomento de la Investigación Científica y Técnica de Excelencia, Subprograma Estatal de Generación de Conocimiento del Ministerio de Economía y Competitividad (Ref: CSO2016-78386-P).
} 


\section{ABSTRACT:}

Culture plays a key role as an engine of social change and in development processes. Now, in cognitive capitalism, the commodity of the immaterial constraints diversity and pluralism, homogenizes imaginaries and annuls popular traditions under the pressure of a hegemonic and totalizing mono-discourse. Southern countries, expressions of minorities or third sector media practices, for example, are silenced or displaced to the background, preventing the development of the poor, the displaced or the different. However, the New Technologies of Information and Communication (NICTs) offer a new scenario where citizens can increase participation and local empowerment, keys to the health of democracy. Thus, the informational capital that the Internet provides in terms of participation and citizen cooperation are essential for development and social change. Now, these processes of appropriation of the NICTs, by the citizens, are mediated by a series of tension or dialectical axes. To transcend these difficulties that the digital environment poses about democracy it is necessary to look for theoretical-methodological models of complex mediation that open up public spaces and allow the local empowerment of citizens. In this sense, a series of action lines are proposed to elaborate communication and culture policies that contribute to the development of an active and participatory digital citizenship.

KEY WORDS:

Culture; Participation; Digital citizenship; Communication policies; NICTs; Development; Social change.

\section{Introducción}

La cultura y la participación ciudadana son esenciales para el desarrollo sostenible. Así lo ha señalado la Asamblea General de las Naciones Unidas con su Agenda para el desarrollo sostenible 2030, marcada en septiembre de 2015, en la que se puso de manifiesto la necesidad de tener en cuenta la cultura en la mayoría de los Objetivos de Desarrollo Sostenible (ODS), incluyendo aquellos centrados en la educación de calidad, las ciudades sostenibles, el medio ambiente, el crecimiento económico, las pautas de consumo y producción sostenibles, las sociedades inclusivas y pacíficas, la igualdad entre géneros o la seguridad alimentaria (Unesco, 2015a).

En un contexto social marcado por la omnipresencia las Nuevas Tecnologías de la Información y la Comunicación (NTIC's) no se puede perder de vista cómo Internet está transformando y desplazando una vez más el lugar de la cultura, como diría Homi K. Bhabha, modificando conceptos como el de ciudadanía y participación, provocando gran impacto sobre la democracia misma (Barber, 1984; Hacker y Van Dijk, 2000; Hagen, 2000; Pal, 1997; Subirats, 2002). El presente ensayo esboza a este respecto, brevemente, algunos de los puntos clave para construir una ciudadanía digital activa y participativa y plantea una serie de propuestas para pensar y elaborar políticas de comunicación y cultura que contribuyan para el desarrollo en la ciberdemocracia. El enfoque adoptado es crítico y se consideran retos y agenda de futuro a plantear por autoridades locales y Tercer Sector, en un tiempo y en una dinámica social marcadas por la presencia de las NTIC's y por la emergencia y dominio de lo que algunos autores convenimos denominar capitalismo cognitivo.

\section{Cultura y desarrollo en el capitalismo cognitivo}

La cultura es en definición de la Real Academia Española un conjunto de modos de vida, costumbres, conocimientos y niveles de desarrollo científico, artístico y económico que conforman los valores, normas y pautas de comportamiento de una colectividad. Por ello la pro- 
ducción, difusión y consumo de bienes culturales presupone el reconocimiento de un derecho común, de una dimensión pública compartida por la ciudadanía.

Si se acepta que todo derecho es una producción cultural sujeta a luchas y conquistas políticas como resultado de un proceso de construcción colectiva y de asignación de valor que da sentido común a la existencia, definiendo los puntos de consenso y producción de la ciudadanía, la comunicación constituye, nadie hoy lo discute, un espacio de traducción de lo cultural que hace posible el habitar humano con los otros, esto es, la propia posibilidad de desarrollo. Es más, «desde el patrimonio cultural a las industrias culturales creativas, la cultura es facilitador y motor de las dimensiones económica, social y ambiental del desarrollo sostenible» (Unesco, 2015a), dado que

la cultura es un componente esencial del desarrollo humano, que constituye una fuente de identidad, innovación y creatividad para las personas y las comunidades y que es un factor importante para la inclusión social y la erradicación de la pobreza al promover el crecimiento económico sostenible y la implicación en los procesos de desarrollo".

Asímismo, la comunicación es un hacer relacional que articula las formas intersubjetivas de conciencia y participación conjunta de los actores sociales como espacio de articulación del cambio social y el desarrollo. Por ello, sin comunicación y cultura ninguna estructura social logra integrarse en un proceso de cambio, condenando al fracaso todo proyecto local de mediación y la posibilidad misma de desarrollo endógeno. La comunicación, como indica Rosa María Alfaro Moreno (2008), hace posible entonces que identidades culturales heterogéneas puedan articularse en un mismo proyecto. De ahí que desde los años setenta del pasado siglo, se vincularan los derechos culturales, y las políticas nacionales de comunicación a las posibilidades de un desarrollo autónomo de los pueblos sometidos a condiciones adversas de modernización.

Toda defensa de los derechos de comunicación ha sido desde entonces ligada a los debates sobre modelos y objetivos de desarrollo, a la discusión, en fin, sobre el modelo de sociedad que representamos y tratamos de proyectar en la práctica. Así, hoy la información y el conocimiento son considerados bienes necesarios para promover el desarrollo. Más aún en un tiempo como el presente en el que la dialéctica de la innovación hace real el principio de «cada uno construye según sus capacidades y el producto se distribuye según las necesidades» en una forma de materialismo que, a decir de Raymond Williams (1980), confirma que la cultura es un estilo de vida, un modo de vida total.

Con la emergencia de la Sociedad de la Información y la omnipresencia de Internet en las relaciones e interacciones sociales y en la política, es preciso volver a pensar críticamente esta cuestión con la diferencia de que la cultura se ha convertido, en el «capitalismo cognitivo» (Blondeau et al., 2004), en un recurso estratégico de las políticas internacionales de desarrollo. Hoy en día las Nuevas Tecnologías de la Información y la Comunicación (NTIC) arrasan con los canales tradicionales de la cultura y sus formas (García Gutiérrez, 2003). De ahí la crítica de Vandana Shiva (2007) a la mercantilización del infoconocimiento y de la

1/ 68/223 Cultura y desarrollo sostenible. Resolución aprobada por la Asamblea General el 20 de diciembre de 2013. Recuperado de https://www.un.org/en/ga/search/view_doc.asp?symbol=A/RES/68/223\&referer=http\%3A//www.un.org/en/ga/68/resolutions.shtml\&Lang=S 
actividad creativa que disminuye o anula la diversidad cultural, capitaliza los saberes tradicionales, las formas no instrumentales de intercambio, el pluralismo cultural y la diversidad y complejidad de toda ecología de comunicación por imposición de una relación lineal hegemónica y totalizadora entre conocimiento público y apropiación privada.

Boris Groys define claramente la cuestión de esta lógica de la economía cultural como «un factor estratégico de los procesos de desarrollo hipermediatizados de nuestra contemporaneidad, donde la innovación se consuma, principalmente, en la forma cultural-económica del intercambio» (2005: 159).

Esto es, la innovación y el desarrollo es resultado sobre todo de la capacidad de combinación tanto en formas y configuraciones, como en contenidos y procesos. Una suerte de dinámica del palimpsesto, de borrado y reescritura de la memoria conservada, como propone Moles, por el que se muda o altera el sentido original, el contexto de procedencia para cumplir una función mercantil.

Un proceso que se podría explicar como la labor del periodismo -cortar y pegarcomo una actividad moderna de montaje y difusión o, en otros términos, como una función valorizadora que distingue el tiempo actual como la era de la copia. Pues la innovación sólo puede operar con aquello que ya está a mano, que posee un valor determinado en la memoria culturalmente valiosa o en el espacio de lo profano, y sólo pretende una modificación de la relación entre esos valores. Luego la innovación es una operación comercial, una mediación (Martín-Barbero,1987; Martín Serrano, 1978) que pone en valor la tradición cultural concediendo dignidad a lo profano, especulando en el plano del signo cultural.

Así, la producción no es más que una determinada forma del uso, mientras que la pura contemplación, es decir, el uso puro, es el que consigue generar nuevos valores. Las culturas se han caracterizado, de hecho, por sus permanentes procesos de adaptaciones creativas. De lo contrario tales culturas no existirían. De ahí la pertinencia de tomar en consideración, siguiendo a Vandana Shiva (2007), la diversidad en términos de igualdad, en términos de política democrática de la representación. Esto es, para empezar a plantearnos en todas sus consecuencias la relación entre NTIC's, cultura y desarrollo debemos primero tomar en cuenta tres principios fundamentales:

- Reciclaje, basado en la devaluación de lo valioso y la revalorización de lo devaluado, bajo la asunción de que toda creación parte de intercambios innovadores previos.

- Sostenibilidad de la ecología cultural, que se traduce en una defensa radical del derecho a la biodiversidad y al desarrollo endógeno de la cultura.

- Equidad creativa, que pone de manifiesto la necesidad de fomentar la creatividad, la fluidez y la igualdad y, sobre todo, evitar la rigidez y las formas de exclusión.

\section{Comunicación, desarrollo democrático y mercantilización de la cultura}

A la hora de reflexionar acerca de la comunicación y el desarrollo de la democracia en la era digital es preciso tener en cuenta el telón de fondo del capitalismo cognitivo. La reestructura- 
ción y transformación que las NTIC's provocan sobre la sociedad, la cultura y la ciudadanía van mucho más allá de una mera apropiación de herramientas digitales. Justamente la cultura se encuentra a día de hoy - como la mayor parte de las actividades sociales - absorbida por la lógica del mercado y la «neoliberalización» de todos los procesos creativos.

Tomar consciencia de esta dinámica es sólo el primer paso para definir los retos democráticos para el desarrollo de los medios de comunicación y las políticas de comunicación que acompañan, gestionan y regulan los sistemas de información digital. Como ha mencionado Sierra Caballero (2016), desde finales de la década de los setenta la noción de desarrollo cultural ha relacionado el campo de la comunicación a necesidades sociales de ámbitos diversos como «la economía, la política o la educación, integrando la creatividad y las identidades plurales en la dinámica constituyente del modelo de reproducción modernizadora, bajo el auspicio fundamentalmente de las Naciones Unidas (ONU)» (Sierra Caballero, 2016: 130). Es más, numerosos programas de organizaciones como la Organización Mundial de la Salud (OMS) o la Organización de las Naciones Unidas para la Alimentación (FAO) han incluido la comunicación en sus objetivos sectoriales y en la base de sus políticas de cooperación internacional.

Estos y otros organismos han tenido siempre una visión instrumental de los medios de comunicación y de la información como una fuente de progreso y crecimiento fundamentalmente económico. Plantear la comunicación desde esta perspectiva mercantilizada deja a un lado elementos que son clave a día de hoy en los contextos digitales: la participación de la ciudadanía, la creatividad social y la innovación por parte de los países del sur o las comunidades indígenas, el intercambio y cooperación entre países, por citar unos pocos. Más aún en las sociedades globalizadas donde la lógica neoliberal imperante coarta e impide el desarrollo de la cultura popular o de los países periféricos, dependientes del mercado global hegemónico, angloamericano y homogeneizante.

Ahora bien, por otra parte, de forma contradictoria, esta misma centralidad de la comunicación y la cultura sirve también - según advierte Yúdice (2002) - como un nuevo fundamento o garantía para exigir derechos en la plaza pública en conflictos interculturales o luchas por el territorio, los recursos o la dominación y privación de medios de expresión y representación cultural. Por ello mismo, se defiende que la comunicación es un instrumento estratégico de cambio social (Arévalo Salinas, Vilar Sastre y García López, 2019; Arévalo Salinas y Farné, 2016; Barranquero Carretero y Sáez Baeza, 2010; Gumucio-Dragon, 2011; Obregón, 2007) y el principal dispositivo de poder en la Sociedad del Conocimiento.

\section{Del «capital social» al «capital informacional», NTIC's y participación}

Uno de los debates centrales en torno a la Sociedad Europea de la Información es la función provisoria de «capital social» que aportan los nuevos medios, tal y como plantea la Informática Comunitaria (IC) (Sánchez Lugo, 2006) o los estudios de politólogos sobre confianza y buenas prácticas de gobernanza en entornos digitales (Van Bavel, Punie y Tuami, 2004)

El concepto de «capital social», acuñado por Pierre Bourdieu (1980) y reinterpretado por James Coleman (1990) y Richard Putman (1993a; 1993b; 1996), designa -en el marco de esta investigación - el vínculo por el que se organiza toda comunidad en función del grado de 
participación, en tanto forma de sociabilidad. Y es que la participación ciudadana está en el corazón de la democracia (Verba, Schlozman y Brady, 1995) y es un indicador de la calidad y madurez de un sistema democrático (Gerodimos, 2010; Gerodimos y Ward, 2007). Es más, la participación fomenta el desarrollo de un buen concepto de ciudadanía que necesariamente implica: compartir responsabilidades, involucrarse con la vida pública o preocuparse por las cuestiones de interés general (Sola-Morales y Hernández-Santaolalla, 2017). Mediante la misma los individuos pueden ejercer la ciudadanía (Marshall y Bottomore, 1998), tomar partido en los asuntos sociales e institucionales (Merino, 1996) y ejercer control sobre el poder que ostentan los representantes (Guillen et al., 2009).

Desde la perspectiva de Putnam el carácter colectivo de la participación y las redes sociales, que forman la comunidad cívica, son esenciales, así como el sentimiento de pertenencia, la cooperación entre miembros y la confianza. El autor cuantifica la participación ciudadana como un indicador de la mayor o menor disposición de capital entre diferentes colectivos de población, atribuyendo la crisis de confianza y la pérdida de autonomía local a múltiples factores que inciden en el aislamiento y desconexión ciudadana, entre ellos, por ejemplo, medios tecnológicos como la televisión o Internet, en la medida que tienden a separar a los miembros de una comunidad dada estableciendo distancias e intereses disímiles contrarios, o al menos nada favorables, a la vida en común.

Otras interpretaciones apuntan, por el contrario, la relevancia de las NTIC's en el desarrollo de experiencias de empoderamiento local. De acuerdo con Van Bavel, Punie y Tuami (2004), las NTIC's tienen un papel cada vez más importante en la creación y asignación de «capital social» por la capacidad que tienen de movilizar recursos materiales, información y conocimiento. Si el «capital social», de acuerdo con Putnam (1996), puede ser definido como una de las características de la organización social vinculada a la cooperación en beneficio mutuo, la confianza y participación cívica, el vínculo con nuevas tecnologías puede plantear algunas problemáticas. De hecho, las redes pueden socavar el capital social, pues el tiempo destinado a la interacción mediada tecnológicamente puede empobrecer las relaciones sociales y la cohesión colectiva limitando la participación ciudadana.

Los portales ciudadanos, por ejemplo, demuestran, sin embargo, que las NTIC's pueden ser catalizadoras de las formas alternativas de cooperación social y acción colectiva, activando y transformando significativamente el capital social, en palabras de Van Bavel et al. (2003) en «capital social interconectado». El grado de interconexión, la extensión y calidad de las redes, el lenguaje de los vínculos, son indicativos, a este respecto, de la calidad y complejidad de la participación.

La apropiación social de las NTIC's apunta, en esta dirección, al pleno desarrollo de la capacidad individual y colectiva de interconectar realidades presentes en el nuevo entorno informativo y mediatizado tecnológicamente desde la estructura cognitiva y los propios mundos de vida para un uso pragmático y creativo de los nuevos ecosistemas de interacción y transformación cultural, en función del contexto inmediato de los actores locales. Este reto no es solo un problema de dotación física de equipamientos e infraestructura pública. Cabe distinguir a este respecto entre «capital social» $y$ «cultivo social» (la cultura y desarrollo de redes ciudadanas articuladas en las comunidades, más allá de las condiciones objetivas o materiales) (Vizer, 2003).

Ambos componentes deben ser considerados en la extensión de las NTIC's para una ciudadanía activa, especialmente el «cultivo social», por cuanto constituye la trama expresiva 
de formaciones de sentido en la vida social, que permite formas organizativas de calidad y complejidad superior, transformando a los actores sociales en agentes activos del cambio del entorno. Justamente, las NTIC's fomentan una información/comunicación sin intermediarios (Rucht, 2004), que permite a los ciudadanos corrientes o activistas crear mini-medios (Carty, 2002) e informar sin tener que depender de los medios convencionales (Woong, 2001), así como trascender fronteras (Wray, 1998; Garrido y Halavais, 2003; Martínez-Torres, 2001), por lo que se podría concluir que la participación se incrementa (Kollock y Smith, 1999) al tiempo que se reducen los costes de creación, organización, participación (Earl y Kimport, 2011; Danitz y Strobel, 1999; Rosenkrands, 2004; Salter, 2003).

La relevancia de la dimensión cultural, o simbólica, así como los útiles comunicativos para la participación local, deriva, como advierte Yúdice (2002), de la transformación de la cultura en un recurso y objeto de reclamo sustitutivo, con frecuencia, de la política y la economía en los procesos de desarrollo territorial. Así, si bien en los años sesenta las políticas locales de desarrollo se centraban en la inversión en capital físico y una década más tarde se descubrió la importancia económica de inversión en las personas, hoy las políticas públicas priorizan la inversión en capital/cultivo social. Esta dimensión de la política de la representación:

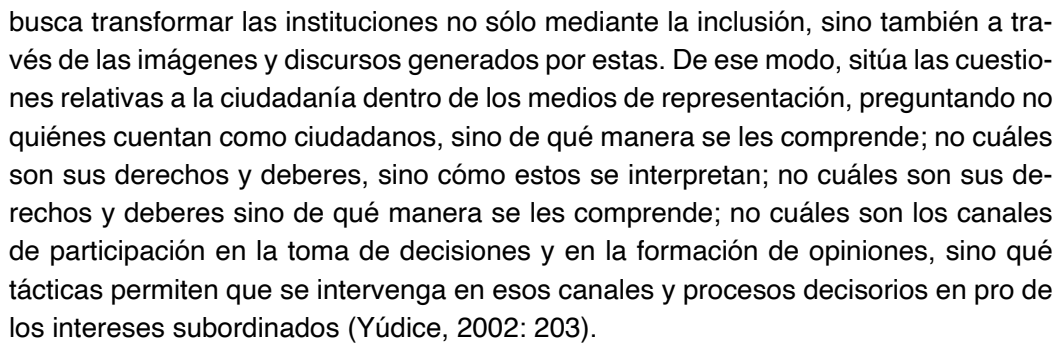

Desde este punto de vista, se puede hablar de diversos niveles de complejidad a la hora de analizar el proceso de apropiación de las NTIC's por parte de los actores sociales. Para ello, un concepto fundamental es el de «capital informacional», más vinculado al trabajo teórico de Pierre Bourdieu o al de Cees Hamelink (2000), que a los planteamientos de Putnam.

El «capital informacional», desde la perspectiva de Hamelink, comprende la financiación de las redes electrónicas, la habilidad técnica para poder manejar sus correspondientes infraestructuras, la capacidad intelectual que permite filtrar y evaluar la información que circula en ellas, así como la motivación para utilizar la información en situaciones sociales.

Ello presupone diferentes condiciones y movimientos en relación con la organización: de la información a la deliberación, de los procesos de consulta y dinamización cultural a la elección y decisión vinculante. La complejidad y posibilidades de la democracia participativa en el uso y gestión de los sistemas avanzados de información ofrece, por ello, diferentes alternativas para el diseño de un entorno inteligente y abierto de interacción.

Hoy, sin embargo, se da la paradoja de que, ante la crisis de la representación y la gobernanza en un contexto de grandes transformaciones provocadas por la globalización, las autoridades locales y en general la Administración, se ven obligadas a reinventar la democracia local y la representación a escala global. Ahora más que nunca, la democracia parece requerir más innovación y creatividad, más participación y autonomía social. En el contenido y tensión de la que es portadora esta paradoja se puede situar la crítica a las deficiencias 
del modelo representativo ante la intensificación a escala geométrica de los procesos de globalización y sus efectos colaterales en el plano local, entre ellos la susodicha desconexión de los ciudadanos, la falta de compromiso cívico o la negación directa a participar de los tradicionales modelos patriarcales de domesticación, claramente inadecuados en la cultura y formas de interacción de la era digital.

La propuesta política de la ciberdemocracia trata, a este respecto, de trascender la participación individual y/o comunitaria de los estudios sobre «capital social» y desarrollo por un modelo teórico-metodológico de la mediación compleja y solidaria que se centre en la dimensión política de la apertura de espacios públicos locales, en la formación ética de la ciudadanía y en el empoderamiento local desde una concepción praxiológica.

Si algún sentido tiene apostar por la participación como principio rector de la democracia y el desarrollo local, es porque se concibe la comunicación, como contexto y horizonte de progreso, para favorecer las relaciones anticipatorias y liberadoras, porque se aspira a promover relaciones de cooperación y formas de ciudadanía activa, porque, en suma, se apuesta por activar las relaciones de confianza y el interés público a partir de los contextos locales y los mundos de vida. El desarrollo social de las NTIC's debe, en este sentido, ajustarse a los objetivos de apropiación social por la comunidad, a las necesidades radicales de expresión y desarrollo cultural de la ciudadanía, así como a los retos económicos-políticos de interés público de la ciudad. Es necesario pensar la participación y las posibilidades abiertas por los nuevos medios digitales como un proceso de construcción colectiva del desarrollo local basado en la cooperación, la organización de redes cívicas y el diseño de los planes de cambio social a partir de la creatividad individual y colectiva de los actores locales y de los ciudadanos mismos.

Por supuesto, este empeño significa ir más allá de facilitar el acceso a la Red. Se trata más bien de procurar experimentar con la creación y autoorganización de la ciudadanía, de acuerdo con los principios de la democracia radical participativa. Ahora bien, esta propuesta plantea problemas en Europa, visto que la participación política y la ciberdemocracia son definidas de acuerdo con la cultura política dominante y los límites jurídicos e institucionales de las formas modernas de ordenamiento político y social. Si las NTIC's pueden fortalecer la capacidad de autogobierno de las comunidades locales superando las diferencias económicas y sociales mediante la contribución a la creación de espacio público y dinámicas de participación igualitarias en los planes de desarrollo local, es a condición de que los movimientos sociales y la sociedad civil organizada lideren la lucha por la defensa de los derechos culturales. Pues sólo las redes críticas de acción colectiva pueden desplegar la capacidad necesaria de movilización y extensión de vínculos sociales que precisa, en este sentido, el desarrollo y la participación con las NTIC's, si de configurar otro espacio público local se trata como nuevo horizonte cognitivo de democratización.

\subsection{EJES DE TENSIÓN EN EL PROCESO DE APROPIACIÓN DE LAS NTIC’S}

Desde el primer Foro Social Mundial (2001), en el que el debate en materia de comunicación fue marginal y disperso, hasta el de 2005 , en el que el tema de la cultura y la comunicación ocupó cuatro de los once espacios temáticos que componían el Foro, en el Tercer Sector se han ido forjando en los últimos años las bases de una filosofía sobre los bienes públicos co- 
munes (cultura, información, comunicación, educación, salud, agua), en tanto que dominios que deberían escapar a la sola lógica del mercado para ser regidos por el principio del servicio público. Tomando como referencia el concepto de «capital informacional», se identifican una serie de tensiones o dialécticas en el proceso de apropiación de las NTIC's por parte de los actores sociales y la ciudadanía:

1) Tensión información vs cultura digital en la era global. La cual implica siguiendo a García Gutiérrez (2003), la confrontación entre la imaginación, el sentido y la lógica de los afectos frente a llamada ingeniería social, en la que los prototipos digitales «farwesternizan» la cultura. A saber, la imposición de la racionalidad instrumental y la tecnocracia frente la socialización comunitaria, ejes fundamentales de la cultura.

2) La dialéctica entre libertad y exclusión. Si bien las redes sociales y bases de datos fomentan el tráfico de la cultura y, de algún modo, potencian la socialización y los vínculos comunitarios, por otro lado, el mercado abierto que fomenta el neoliberalismo genera lógicas de exclusión: expulsa a numerosos sectores y agentes culturales y estratifica los consumos. Como mecanismo de resistencia frente a esta lógica surgen formas de apropiación común, que son tachadas por las industrias culturales hegemónicas como piratería -copia, reproducción o uso indebido sin expresa autorización de sus titulares, de cualquier obra protegida como propiedad intelectual-. «La cuestión en este punto de la libertad de circulación y acceso y de la criminalización de las reapropiaciones culturales sería definir qué es un uso indebido ¿Quién y cómo se establece qué es lo debido y legal?» (Sierra Caballero, 2016: 153).

Es más, tal y como declara la Carta de la Comunicación de los Pueblos ${ }^{2}$ y la declaración del Movimiento Internacional por una Voz Popular en los Medios y Comunicaciones en el Siglo xxl3 , acceder a las NTIC's es un derecho humano fundamental. Más hoy en día cuando aún existen problemáticas con el acceso universal a una tecnología que debiera estar al alcance de todos. La defensa de estos derechos, de acceso y participación, es fundamental y está en la base de un modelo de desarrollo que permita trascender los marcos excluyentes de la mercantilización imperante en las sociedades actuales.

3) La dialéctica entre el interés público y la concentración. La concentración de la industria cultural y la ausencia de diversidad provocada por las limitaciones al pluralismo ideológico y social son importantes lacras que impiden el desarrollo. Esta lógica ha boicoteado y limitado voces, anulado imaginarios y narrativas, borrado o desdibujado memoria, ritos y tradiciones plurales compartidas, basadas en la riqueza y la diversidad cultural. Es más, esta concentración orquestada por las corporaciones transnacionales, con un diseño basado en la homogeneización de ecosistemas culturales, es la norma implícita y claramente manifiesta de instituciones como la Unión Europea o diversos organismos que se amparan en el comercio internacional para someter y enajenar derechos de dominio público de economías locales a nivel mundial, tal y como han criticado de manera reiterada María Mies y Vandana Shiva (2016).

2/ Véase: http://www.pccharter.net/

3/ Véase: http://www.comunica.org/v21/statement.htm 
Justamente la defensa del dominio público es una opción que va más allá de lo estratégico. Es necesario denunciar la concentración de canales, recursos simbólicos y contenidos por parte de las grandes compañías multimedia, en diferentes niveles del proceso de mercantilización de la nueva economía de la cultura (en la producción, tanto como en la distribución o fijación en soportes, y como consecuencia, también, como resultado, en la determinación de las formas de consumo cultural) (Sierra Caballero, 2016).

4) La dialéctica entre la transparencia y la opacidad. Esta confrontación pone de manifiesto la falta transparencia y la preponderancia de «una cultura dominada por los lobbys multinacionales, la estandarización del derecho privado y la prevalencia de exigencias y acuerdos bilaterales que marcan la regulación nacional y aun supranacional de los espacios de integración económica» (Sierra Caballero, 2016: 155).

5) La dialéctica entre la creatividad y la inteligencia social general y la apropiación individual. Aquí se manifiestan algunas dificultades como la gestión del conocimiento, la apropiación individual o colectiva de las tradiciones y el folclore y los procesos de identificación que se dan en torno al desarrollo cultural. Organismos internacionales como la UE plantean calidad, innovación y competitividad como bases del desarrollo, dejando a un lado y anulando si cabe cualquier forma de participación activa, cualquier forma de socialización creativa. Desde estos organismos se piensa «el desarrollo, como la tradición de la difusión de innovaciones, desde una lógica metodológicamente individualista, lo que no deja de ser contradictorio, o cuando menos paradójico en la era de las multitudes inteligentes, de socialización de los medios de producción y generación de riqueza» (Sierra Caballero, 2016: 156), en sentido material y simbólico, especialmente en virtud del lenguaje de los vínculos.

\section{Hacia una política de comunicación participativa y una ciudadanía digital}

Las NTIC's pueden aportar sentido y dirección como parte de una estrategia de comunicación más amplia en la que combinar acciones políticas públicas offline y online. Los procesos de deliberación, organización, y movilización ciudadana se encuentran hoy en día embebidos por el impacto de la tecnología, motivo por el cual es necesario elaborar políticas de comunicación que sean capaces de contribuir a los procesos de comprensión y participación de la ciudadanía en entornos digitales.

En este contexto social y político transmediado digitalmente (Gerbaudo, 2012) es preciso trascender la instrumentalización preponderante de la cultura, causada por el neoliberalismo dominante y frenar el individualismo exacerbado fomentado por el consumismo excesivo. Estas lacras impiden ver más allá de la pantalla o el espejo y construir un verdadero sentido de ciudadanía activa y cívica. Vivimos en una época donde las redes sociales utilizadas en el espacio de lo común son esencialmente virtuales y las interacciones culturales están más que nunca marcadas por lógicas opresivas que anulan las características básicas de lo cultural y lo comunitario, esencias del ser humano como ser comunicativo que vive mediado por símbolos, ritos, tradiciones, narración, imaginación y memoria (Cassirer, 1963; 1976; Geertz, 1981; Duch 2002a; 2002b). 
Así, se plantea necesario reivindicar una serie de líneas de actuación básicas para el desarrollo de una política de comunicación que incorpore un buen concepto de ciudadanía digital y que tenga presente los siguientes elementos:

6) El monopolio de la propiedad, el legado cultural y el problema de los intermediarios. Como es sabido todo artista, creador, intérprete o traductor reutiliza el legado cultural y añade, elimina y transforma algo que ya existía. El derecho a la propiedad exclusiva carece de más sentido aún en una sociedad digital donde el corta-pega es la base de la comunicación y el intercambio. Las políticas públicas que priorizan a día de hoy los derechos de autor y legitiman la apropiación privada están a un tiempo fomentando «la explotación, el uso, la comercialización y dominio privado de los intermediarios, llámese autor, empresa periodística o buscador como es el caso disputado de Google» (Sierra Caballero, 2017: 160).

7) La conformación del espacio y el dominio público. En los entornos digitales el problema del derecho de propiedad intelectual es una cuestión de límites (Smiers, 2006). Es necesario en primer lugar reconocer el dominio público colectivo y respetar la comunidad, así como las libertades públicas y los derechos colectivos, base de una cultura para el desarrollo que sea balanceada y armónica. Ahora bien, en un contexto en el que dominan las lógicas impuestas por los organismos transnacionales y el mercado, es difícil dar espacio a la memoria, la comunidad o la identidad cultural, elementos clave en el desarrollo de un buen concepto de ciudadanía digital.

8) La libertad de flujos de información y de acceso. En el ciberespacio se desarrollan innumerables prácticas culturales ajenas al mercado. Es por ello preciso proponer un modelo de regulación adaptado a las nuevas prácticas. El derecho al libre acceso a la información - como se plantea en la Declaración de San José- debe ser entendido como derecho a la cultura y al desarrollo, que afirme y, sobre todo, reconozca el carácter comunitario y público del arte y la cultura, más allá de la dependencia tecnológica y de la obsolescencia de la propiedad intelectual (Sierra Caballero, 2017).

9) La creación y la recreación como obra social. En la era digital donde las multitudes conectadas crean y cooperan, el concepto de artista o la autoría propia de la llustración ha perdido gran parte de su sentido. Aquí se plantea, por ende, una lectura transversal comunitarista y socializadora que permita dialogar, criticar y participar integrando modelos sociales y colectivos de gestión de la red frente a monopolios como la SGAE o Google (Sierra Caballero, 2017).

10) La cooperación como base de la acción social. En contra de la racionalidad tecnológica mercantil se reivindican los nuevos modelos de cooperación social e internacional entre actores sociales y culturales que apuestan por los procesos participativos y autogestionarios. La era Google refuerza el principio de división internacional del trabajo cultural (Miller y Yúdice, 2002), motivo por el cual es preciso tomar como referentes indicadores de calidad e innovación que vayan más allá de la política científico-técnica neoliberal vendida al imperativo neoliberal.

11) La ecología cultural sostenible. En un contexto donde predomina la aceleración de flujos (Harvey, 1998) es preciso fomentar la cultura slow - por contraposición a la extrema aceleración cultural que provoca el capitalismo (Agger, 2004; Gane, 2006) - tal y como han sugerido Virilio (1986) o Gane (2006). Ello implica -en palabras de Sierra Caballero: 
Una visión inmanentista de la información y el conocimiento, del desarrollo y la cultura, a partir de procesos endógenos de despliegue lento de las culturas populares posiocionándonos en los tiempos de transformación carnavalesca, en la escucha atenta de las comunidades, de sus voces e imaginarios, en el tiempo de los caracoles, frente a la velocidad de escape de las máquinas de procesamiento de información (Sierra Caballero, 2016: 165).

\section{Discusión y conclusiones}

El paso a este nivel de reflexividad implica una visión política de la comunicación y las NTIC's en consonancia con el compromiso cívico de incrementar la participación ciudadana. Ahora bien, la apropiación individualizada de las NTIC's fragmenta y atomiza a los actores sociales en gran medida. El acceso y la participación restringida -por falta de recursos económicos y simbólicos- en las fases de producción y distribución no son el único problema. Justamente el desarrollo de una participación activa se encuentra particularmente debilitado: el derecho a la participación no está definido entre derechos y deberes por el Estado Moderno (Sierra Caballero, 2016). Tampoco la desafección ciudadana (Pharr y Putnam, 2000; Pharr, Putnam y Dalton, 2000) y la abstención electoral masiva a nivel mundial (Carlin, 2006; O'Toole et al, 2003; Phelps, 2006; Skelton and Valentine, 2003; Wiseman, 2006), la crisis de legitimidad del sistema representativo (Alcántara, 1994; Bromley, Curtice y Seyd, 2004; Crozier, Huntington y Watanuki, 1975; Shields, 2006) o la corrupción que asola muchas democracias modernas (Barreiro y Sánchez-Cuenca, 2000; Pérez Gálvez y López Fernández, 2014; Ordóñez, 2012) impiden el desarrollo de una ciudadanía digital activa y ponen en peligro la participación.

El asunto se complica si se añade la arista tecnológica, pues la ciudadanía digital carece de reconocimiento jurídico (Sierra Caballero, 2016) y muchas de las iniciativas virtuales llevadas a cabo por instituciones y gobiernos parecen atender más al voluntarismo político que a una pauta o estrategia delimitada. Ante el vacío legal es preciso, por tanto, plantear la exigencia de reconocer el derecho a comunicarse, expresarse y formar redes ciudadanas colectivas en los entornos digitales.

Ahora bien, la dificultad que se plantea es cómo evaluar las políticas públicas o qué indicadores definir para garantizar la participación cívica y el desarrollo mediante las NTIC's. Como ha indicado Sierra Caballero:

\footnotetext{
El problema de las políticas locales debería ser cómo evaluar y definir la participación como apuesta por una democracia radical y pluralista, máxime cuando la definición de la cultura como recurso viene condicionada por las políticas internacionales de desarrollo en la gestión, almacenamiento, distribución y organización del acceso a los bienes simbólicos, sujeta a las condiciones de circulación y valorización transnacionales del capitalismo (Sierra Caballero, 2016: 166).
}

En este sentido, sería necesario pensar la democracia más allá de la racionalidad tecnocrática y la eficiencia informacional atendiendo al impacto de las NTIC's sobre la participación ciudadana, aprovechando las ventajas que los entornos digitales aportan. Bien sea dando un mayor énfasis a la cantidad (la participación aumenta) o a la cualidad (la participación cambia), lo cierto es que hoy en día hay sobradas pruebas de que la flexibilidad y fluidez 
provocadas por el capitalismo tardío (Castells, 2001; 2003; Manovich, 2005; Scolari, 2008) se materializan en nuevas formas de participar mediadas por la tecnología. $Y$ es que las formas de información, deliberación o asistencia mediadas por la tecnología pueden amplificar o catalizar la participación de los ciudadanos o de un movimiento social determinado transformando su lógica interna o su manera de captar seguidores, por ejemplo. Por ello es necesario cuestionar «la noción misma de ciudadanía y el marco jurídico de la participación en el Estado social de derecho en el marco de evolución del Estado-nación al Estado móvil, que prefigura el Capitalismo Cognitivo» (Sierra Caballero, 2016: 167).

En definitiva, reflexionar sobre la ciudadanía digital hoy implica necesariamente tener en cuenta el impacto de la NTIC's sobre la participación, pero sobre todo, reflexionar sobre las mediaciones y las prácticas culturales que se dan en el entorno digital. Y es que si queremos que las NTIC's favorezcan el desarrollo sostenible, como plantea la Unesco en su Agenda para el Desarrollo Sostenible 2030 (Unesco, 2015b) es preciso plantear una participación activa en la que los ciudadanos puedan interactuar, cooperar y organizar redes cívicas digitalmente y, sobre todo, diseñar la organización del cambio social mediante la comunicación y la cultura como matrices esenciales del ecosistema mediático.

\section{Bibliografía}

AGGER, Ben (1991). Fast capitalism. Urbana. University of Illinois Press.

AGGER, Ben (2004). Speeding up fast capitalism. Boulder, CO: Paradigm.

ALCÁNTARA, Manuel (1994). Gobernabilidad, crisis y cambio. Madrid: Centro de Estudios Constitucionales.

ALFARO MORENO, Rosa María (2008). "Ciudadanos y culturas mediáticas: ocultos en la formalidad democrática». Mediaciones sociales, 3, 351-391.

ARÉVALO SALINAS, Alex Iván y FARNÉ, Alessandra (2016). «Comunicación y cambio social. Un análisis desde la investigación centrado en el periodismo». Cultura, Lenguaje y Representación, 15, 11-19. DOI: 10.6035/clr.2016.15.1

ARÉVALO SALINAS, Alex Iván, VILAR SASTRE, Griselda y GARCÍA LÓPEZ, Marcial (2019). Comunicación y cambio social. Barcelona: Tirant lo Blanch.

BARBER, Benjamin (1984). Strong democracy: Participatory politics for a new age. Berkeley: University of California Press.

BARRANQUERO CARRETERO, Alejandro y SÁEZ BAEZA, Chiara (2010). “Comunicación alternativa y comunicación para el Cambio social democrático: Sujetos y objetos invisibles en la enseñanza de las Teorías de la comunicación». En Congreso internacional AE-IC Malaga Comunicación y desarrollo en la era digital. Universidad Autónoma de Barcelona.

BARREIRO, Belén y SÁNCHEZ-CUENCA, Ignacio (2000), «Las consecuencias electorales de la corrupción». Historia y política: Ideas, procesos y movimientos sociales, 4, 69-92.

BLONDEAU, Olivier, WHITEFORD, Nick D., VERCELLONE, Carlo, KYROU, Ariel, CORSANI, Antonella, RULLANI, Enzo, MOULIER BOUTANG, Yann y LAZZARATO, Maurizio (2004). Capitalismo cognitivo, propiedad intelectual y creación colectiva. Madrid: Traficantes de Sueños. Recuperado de https://www.traficantes.net/sites/default/files/pdfs/Capitalismo\%20cognitivo-TdS.pdf

BLUMLER, Jay G. y COLEMAN, Stephen (2001). Realising democracy online. A civic commons in cyberspace. IPPR/Citizens online research publications, 2. Recuperado de http:www.ippr.org.uk 
BOURDIEU, Pierre (1980). «Le capital social». Actes de la Recherche en Sciences Sociales, 31(1), 2-3.

Recuperado de http:www.persee.fr/doc/arss_0335-5322_1980_num_31_1_2069

BROMLEY, Catherine, CURTICE, John y SEYD, Ben (2004). Is Britain facing a crisis of democracy? London: UCL Constitution Unit.

BURGELMAN, Jean C. (2003). «A new paradigm for eGovernment». IPTS Report,74, 4-10.

CARLIN, Ryan E. (2006). «The decline of citizen participation in electoral politics in post-authoritarian Chile». Democratization, 13(4), 632-651. DOI: 10.1080/13510340600791921

CARTY, Victoria (2002). «Technology and counter-hegemonic movements: The case of Nike Corporation». Social Movement Studies, 1(2), 129-146. DOI: 10.1080/1474283022000010646

CASSIRER, Ernst (1963). Antropología filosófica: Introducción a una filosofía de la cultura. México: Fondo de Cultura Económica.

CASSIRER, Ernst (1976). Filosofía de las formas simbólicas. Fenomenología del reconocimiento, Vol. 3. México: Fondo de Cultura Económica.

CASTELLS, Manuel (2003). La era de la información: economía, sociedad y cultura. 2. El poder de la identidad. Madrid: Siglo XXI.

CASTELLS, Manuel (2001). La galaxia Internet. Reflexiones sobre Internet, empresa y sociedad. Barcelona: DeBolsillo.

CENTENO, C., VAN BAVEL, R., y BURGELMAN, J.C. (2004). eGovernment in the EU in the next decade: The vision and key clallenges. Sevilla. IPTS/European Commission.

COLEMAN, James S. (1990). Foundations of social theory. Cambridge: Belknap Press.

COMISIÓN EUROPEA (2004). Challenges for the European Information Society beyond 2005. 19 November.

CROZIER, Michael J., HUNTINGTON, Samuel P. y WATANUKI, Joji (1975). The crisis of democracy. Report on the governability of democracies to the trilateral Commission. New York, NY: New York University Press.

DANITZ, Tiffany y STROBEL, Warren P. (1999). «The Internet's impact on activism: the case of Burma». Studies in Conflict and Terrorism, 22(3), 257-269. DOI: 10.1080/105761099265766

DOWNING, John D. H. (2001). Radical media. Rebellious communication and social movements. Londres: Sage.

DUCH, Lluís (2002a). Mito, interpretación y cultura. Barcelona: Herder Editorial.

DUCH, Lluís (2002b). Antropología de la vida cotidiana. Simbolismo y salud. Madrid: Editorial Trotta.

EARL, Jennifer y KIMPORT, Katrina (2011). Digitally enabled social change. Activism in the Internet age. Cambridge, Massachusetts: The MIT Press.

EUROPEAN COMMISSION (2003). Communication from the Commission to the Council, the European Parliament, the European Economic and Social Committee and the Committee of the Regions The Role of eGovernment for Europe's Future. COM (2003) 567 [SEC(2003) 1038].

FERNÁNDEZ BEAUMONT, José (2004). «Una sociedad de la comunicación construida sobre las buenas prácticas». Telos, 63.

FINQUELIEVICH, Susana (Coord.) (2005). Desarrollo local en la sociedad de la información. Municipios e Internet. Buenos Aires: La Crujía.

FRISSEN, V. (2003). «ICTs, civil society and local/global trends in civil participation». Taller ICTS and Social Capital in the Knowledge Society. Sevilla: IPTS.

GANE, Nicholas (2006). "Speed up or slow down? Social theory in the information age». Information, Communication \& Society, 9(1), 20-38. DOI: 10.1080/13691180500519282 
GARCÍA GUTIÉRREZ, Antonio (2003). «La política europea de la memoria. Una evaluación paraconsistente». El rapto de Europa: crítica de la cultura, 3, 71-82.

GARRIDO, Maria y HALAVAIS, Alexander (2003). «Mapping networks of support for the zapatista Movement: Applying Social-Networks Analysis to Study Contemporary Social movements». En: McCaughey, Martha y Ayers, Michel (Eds.). Cyberactivism: Online activism in theory and practice. New York: Routledge, págs. 165-184.

GEERTZ, Clifford (1981). La interpretación de las culturas. Barcelona: Editorial Gedisa.

GERBAUDO, Paolo (2012). Tweets and the streets: Social media and contemporary activism. London: Pluto Press.

GERODIMOS, Roman (2010). New media, new citizens: The terms and conditions of online youth civic engagement. PhD Thesis, Bournemouth University, UK.

GERODIMOS, Roman y WARD, Janelle (2007). "Rethinking online youth civic engagement: Reflections on web content analysis». En Loader, Brian D. (Ed.). Young citizens in the digital age: Political engagement, young people and new media. London: Routledge, págs. 114-126.

GROYS, Boris (2005). Sobre lo nuevo. Valencia. Pre-textos.

GUILLÉN, Amalia, SÁENZ, Karla, BADII, Mohammad H. y CASTILLO, Jorge (2009). "Origen, espacio y niveles de participación ciudadana». Daena: International Journal of Good Conscience, 4(1), 179-193.

GUMUCIO-DAGRON, Alfonso (2011). "Comunicación para el cambio social: clave del desarrollo participativo». Signo y Pensamiento, 30(58), 26-39.

HACKER, Kenneth L. y VAN DIJK, Jan (Eds.) (2000). Digital democracy: Issues of theory and practice. London: SAGE.

HAGEN, Martin (2000). «Digital democracy and political systems». En Hacker, Kenneth L. y Van Dijk, Jan (Eds.). Digital democracy: Issues of theory and practice. London: Sage, págs. 54-70.

HAMELINK, Cees (2000). The ethics of cyberspace. London: Sage.

HARVEY, David (1998). La condición de la postmodernidad. Buenos Aires: Amorrourtu.

KOLLOCK, Peter y SMITH, Marc (1999). «The promise and the peril of social action in cyberspace». En: Kollock, Peter y Smith, Marc (Eds.). Communities in cyberspace. London: Routledge, págs. 243-263.

LEITNER, Christine (2003). «eGovernment in Europe: The state of affaire». Eipascope, 3, 37-38.

LÉVY, Pierre (2002). Ciberdemocracia. Ensayo sobre filosofía política. Barcelona: Editorial UOC.

MANOVICH, Lev (2005). El lenguaje de los nuevos medios de comunicación. La imagen en la era digital.

Barcelona: Paidós.

MARSHALL, T. H. y BOTTOMORE, Tom (1998). Ciudadanía y clase social. Madrid: Alianza Editorial.

MARTÍN-BARBERO, Jesús (1987). De los medios a las mediaciones. Gustavo Gili, Barcelona.

MARTÍN SERRANO, Manuel (1978). La mediación social. Madrid: Akal.

MARTÍNEZ-TORRES, María Elena (2001). «Civil society, the Internet, and the Zapatistas». Peace

Review, 13(3), 347-355. DOI: 10.1080/13668800120079045

MERINO, Mauricio (1996). La participación ciudadana en la democracia. Quito: CEPAL.

MIES, Maria y SHIVA, Vandana (2016). Ecofeminismo. Barcelona: Icaria.

MILLER, Toby y YÚDICE, George (2002). Cultural Policy. London: Sage.

NAVARRO, Vicente (2003). "Crítica del concepto de Capital Social». Sistema. Revista de Ciencias Sociales, 172, 27-36.

OBREGÓN, Rafael (2007). Comunicación, desarrollo y cambio social. Lecciones del Portal de la Comunicación. INCOM, UAB. 
ORDÓÑEZ, David (2012), «La financiación de los partidos políticos en España: corrupción y deslegitimación». Estudios. Revista de Pensamiento Libertario, 2(2), 19-26.

O'TOOLE, Therese, LISTER, Michael, MARSH, Dave, JONES, Su y McDONAGH, Alex (2003). «Tuning out or left out? Participation and non-participation among young people». Contemporary Politics, 9(1), 45-61. DOI: 10.1080/1356977032000072477

PAL, Leslie A. (1997). «Virtual policy networks: The Internet as a model of contemporary governance?». Internet Society. Recuperado de https://www.isoc.org/inet97/proceedings/G7/G7_1.HTM

PÉREZ GÁLVEZ, Juan Francisco y LÓPEZ FERNÁNDEZ, Francisco Javier (2014). «La credibilidad del sistema democrático español: partidos políticos y corrupción». Revista de Derecho Público, 80, 121-142. DOI: 10.5354/0719-5249.2014.33322

PHARR, Susan J. y PUTNAM, Robert D. (Eds.) (2000). Disaffected democracies? What's troubling the trilateral countries? Princeton, NJ: Princeton University Press.

PHARR, Susan J., PUTNAM, Robert D. y DALTON, Russell J. (2000). «A quarter-century of declinig confidence». Journal of Democracy, 11(2), 5-25.

PHELPS, Edward (2006). "Young citizens and declining electoral turnout: Trends and explanations». En Annual conference The Elections, Public Opinion and Parties (EPOP). Nottingham University.

PUTNAM, Robert D. (1993a). Making democracy work: Civic traditions in modern Italy. Princeton, NJ, Princeton University Press.

PUTNAM, Robert D. (1993b). «The prosperous community: Social capital and public life». The American Prospect, 13, 35-42.

PUTNAM, Robert D. (1996). «The strange disappearance of civic America». Policy: A Journal of Public Policy and Ideas, 12(1), 3-15.

ROSENKRANDS, Jacob (2004). «Politicizing homo economicus: Analysis of anti-corporate websites». En Van de Donk, Wim, Loader, Brian D., Nixon, Paul G y Rucht, Dieter (Eds.). Cyberprotest: New media, citizens, and social movements. New York: Routledge, págs. 49-68.

RUCHT, Dieter (2004). "Movement allies, adversaries, and third parties». En: Snow, David A., Soule, Sarah A. y Kriesi, Hanspeter (Eds.). The Blackwell Companion to social movements. Oxford: Blackwell Publishing, págs. 197-216.

SALTER, Lee (2003). «Democracy, new social movements, and the Internet: A Habermasian analysis».

En: McCaughey, Martha y Ayers, Michael (Eds.). Cyberactivism: Online activism in theory and practice. New York: Routledge, págs. 117-144.

SÁNCHEZ LUGO, José (2006). "Informática comunitaria y la sociedad de la información en Puerto Rico». Acceso: Revista Puertorriqueña de Bibliotecología y Documentación, 8, 1-16.

SANTOS, Boaventura de Sousa (2003). Democratizar a democracia. Os caminhos da democracia participativa, Río de Janeiro: Civilizaçao Brasileira.

SCOLARI, Carlos A. (2008). Hipermediaciones. Elementos para una Teoría de la Comunicación Digital Interactiva. Barcelona: Gedisa.

SHIELDS, J. G. (2006). «Political representation in France: A crisis of democracy?». Parliamentary Affairs, 59 (1), 118-137. DOI: 10.1093/pa/gsj013

SHIVA, Vandana (2007). Las nuevas guerras de la globalización. Madrid: Popular.

SIERRA CABALLERO, Francisco (2017). «La comunicación del conocimiento desde la perspectiva de la preservación del patrimonio cultural y la memoria histórica en el contexto de América Latina». En Pereira, José Miguel (Ed.). Comunicación, lenguajes, TIC e interculturalidad. Cátedra Unesco de Comunicación. Bogotá, D. C.: Pontificia Universidad Javeriana, págs. 145-168. 
SIERRA CABALLERO, Francisco (Coord.) (2016). Capitalismo cognitivo y economía social del conocimiento. La lucha por el código. Quito: Ediciones Ciespal.

SIERRA CABALLERO, Francisco (2006a). Políticas de comunicación y educación. Crítica y desarrollo de la Sociedad del Conocimiento. Barcelona: Gedisa.

SIERRA CABALLERO, Francisco (2006b). Final Report: New Information Technologies, participation and active citizenship. URBACT NETWORK CITIZ@MOVE, Urbact Secretariat, European Commission.

SIERRA CABALLERO, Francisco (2006c). "Nouvelles technologies, participation citoyenne et développement local. Une perspective critique du changement social». Actes du Colloque International Démocratie Participative en Europe, Laboratoire dé Études et de Recherches Appliquées en Sciences Sociales, IUT, Université de Toulouse.

SKELTON, Tracey y VALENTINE, Gill (2003). «Political participation, political action and political identities: young d/deaf people's perspectives». Space and Polity, 7(2), 117-134. DOI: $10.1080 / 1356257032000133892$

SMIERS, Joost (2006). Un mundo sin copyright. Artes y medios en la globalización. Barcelona: Gedisa.

SOLA-MORALES, Salomé y HERNÁNDEZ-SANTAOLALLA, Víctor (2017). «Abstención política y nuevas formas de participación política de los jóvenes: Análisis comparativo entre Chile y España». Revista Latina de Comunicación Social, 72, 629-648. DOI: 10.4185/RLCS-2017-1183

SUBIRATS, Joan (2002). «Los dilemas de una relación inevitable. Innovación democrática y tecnologías de la información y de la comunicación». En Cairo Carou, Heriberto (Ed.). Democracia digital. Límites y oportunidades. Madrid: Trotta, págs. 89-113.

UNESCO (2015a). Cultura para el desarrollo sostenible. Recuperado de https://es.unesco.org/themes/ cultura-desarrollo-sostenible

UNESCO (2015b). Agenda para el desarrollo sostenible. Recuperado de https://www.un.org/ sustainabledevelopment/es/

VAN BAVEL, René, PUNIE, Yves, BURGELMAN, Jean-Claude, TUOMI, Ilkka y CLEMENTS, Bernard (2003). ICTs and social capital in the Knowledge Society. Technical Report EUR 21064. European Commission. Joint Research Centre. Institute for Prospective Technological. Employment Workshop IPTS, Sevilla. 3-4 November 2003.

VAN BAVEL, René, PUNIE, Yves, TUOMI, Ilkka (2004). «Cambios en el capital social, posibilidades por las TIC». Institute for Prospective Technological, 85.

VERBA, Sidney, SCHLOZMAN, Kay L. y BRADY, Henry (1995). Voice and equality. Civic voluntarism in American politics. Cambridge, MA: Harvard University Press.

VIRILIO, Paul (1986). Speed and politics, An essay on dromology. New York: Semiotext(e).

VIZER, Eduardo (2003). La trama invisible de la vida social. Buenos Aires: La Crujía.

WISEMAN, Nelson (2006). «Get out the vote-not: Increasing effort, declining turnout». Policy Options, 27(2), 18-23.

WILLIAMS, Raymond (1980). Marxismo y literatura. Barcelona. Península.

WILLIAMS, Raymond (1982). Cultura, sociología de la comunicación y el arte. Barcelona. Paidós.

WOONG, Loong (2001). «The Internet and social change in Asia». Peace Review, 13(3), 381-387.

WRAY, Stefan (1998). «Electronic civil disobedience and the World Wide Web of hacktivism: A mapping of extra parliamentarian direction action net politics». Paper presented at the World Wide Web and Contemporary Cultural Theory Conference, Drake University, Des Moines.

YÚDICE, George (2002). El recurso de la cultura. Usos de la cultura en la era global. Barcelona: Gedisa. 